\title{
Aldo Rossi et le temps suspendu
}

\section{Can Onaner}

\section{(Q) OpenEdition \\ Journals}

Édition électronique

URL : http://journals.openedition.org/crau/500

DOI : $10.4000 /$ crau. 500

ISSN : 2547-5746

\section{Éditeur}

Éditions du patrimoine

\section{Édition imprimée}

Date de publication : 1 septembre 2013

Pagination : 71-84

ISBN : 978-2-7577-0109-6

ISSN : $1296-4077$

Référence électronique

Can Onaner, «Aldo Rossi et le temps suspendu », Les Cahiers de la recherche architecturale et urbaine [En ligne], 28 | 2013, mis en ligne le 12 septembre 2017, consulté le 19 avril 2019. URL : http:// journals.openedition.org/crau/500; DOI : 10.4000/crau.500 
Les termes "immobilité " et « suspension » apparaissent à plusieurs reprises dans Autobiographie scientifique, sans être théorisés par Aldo Rossi. L'article livre une réflexion autour de la notion de suspens, en la définissant comme un « procédé analogique de glissement du sens ", une " suspension » de la signification des choses. Il s'agit de donner à voir une suspension qui agit dans l'existence même du sujet créateur : substituer les mécanismes artificiels de "l'attente" à l'entropie "naturelle " de la matière, substituer un suspens esthétique à la mort matérielle. Le suspens sert aussi à questionner une vision progressiste de I'histoire de l'architecture en recherchant la nouveauté dans une " compulsion de répétition $»$.

\section{Aldo Rossi et le temps suspendu}

CAN ONANER

«Dans la photographie, l'immobilisation du Temps ne se donne que sous un mode excessif, monstrueux : le Temps est engorgé (d'où le rapport avec le tableau vivant, dont le prototype est l'endormissement de la Belle au Bois dormant). Que la Photo soit «moderne», mêlée à notre quotidienneté la plus brûlante, n'empêche pas qu'il $y$ ait en elle comme un point énigmatique d'inactualité, une stase étrange, l'essence même de l'arrêt ${ }^{1}$. »

Depuis quelques années, dans les médias de l'architecture, comme dans les productions des écoles européennes, nous assistons à la récurrence de formes monolithiques, hors d'échelle, coupées de leurs contextes physiques et temporels, comme suspendues dans une temporalité anhistorique. Que ce soit à travers les formes étranges et décontextualisées de Valerio Olgiati ou les formes monumentales historiquement référencées chez Dogma, Office ou Baukuh, nous assistons à l'apparition d'images fortes, mais énigmatiques. L'expression de ces monolithes égarés semble interrompre

1. Roland Barthes, La chambre claire, Paris, Éditions de l'Étoile, Gallimard, Seuil, 1980, p. 142. 
le mouvement qui nous environne. Ils exposent leurs formes isolées, esseulées et inactuelles, et viennent en contrepied des nouvelles réflexions autour de la mobilité, de l'énergie durable, ou encore des nouvelles technologies de conception et de production.

L'exposition à Archilab intitulée Monolithe et architecture en suspens, témoigne de cet intérêt récent en cherchant à développer une généalogie historique de cette tentative de suspension qui semble innée à I'architecture ${ }^{2}$. D'autres expositions, dans un champ davantage grand public, confirment cette sensibilité pour ce qui semblerait paradoxalement tenir de I'inactuel : I'exposition Architectures de papier et son atelier Villes suspendues à la Cité de l'architecture et du patrimoine, et l'exposition Rêve de monuments, une exposition entre architecture réelle et architecture imaginaire, à la Conciergerie de Paris, ont au moins un aspect en commun : leurs deux affiches donnent à voir une maquette d'architecture suspendue dans l'air ${ }^{3}$. Plus encore que cette suspension physique, ces deux maquettes représentent des bâtiments temporellement suspendus, détachés de leurs contextes historiques et fondamentalement inactuels.

Parallèlement à ces architectures en suspens, nous observons un regain d'intérêt récent pour les recherches des architectes italiens néo-rationalistes des années 1960 et 1970. Preuve en est la récente et très belle exposition à Beaubourg autour de la Tendenza et de sa personnalité phare Aldo Rossi, mettant à l'ordre du jour la richesse plastique et théorique d'une recherche à la fois collective et diverse ${ }^{4}$. Force est de remarquer que cet intérêt renouvelé semble moins porter sur le discours structuraliste autour de la construction de la ville ${ }^{5}$, que sur la poésie particulière d'un langage architectural à la fois froid et monumental, austère et étrange, empruntant aussi bien au classicisme architectural qu'à la peinture métaphysique d'un De Chirico : une sensibilité qui est au cœur même de ce que nous chercherons par la suite à expliciter à travers la notion de « suspens architectural ».

Nous pouvons nous interroger devant le resurgissement de formes néo-rationalistes en référence aux membres de la Tendenza, dans les écoles d'architecture et dans des revues comme San Rocco et CityVision ${ }^{6}$. Nous pouvons aussi nous étonner de l'intérêt renouvelé, chez certains architectes, pour un imaginaire classique et pour des formes primitives et titanesques semblant provenir d'un passé immémorial, à une époque où les nouvelles technologies de conception et les fantasmes robotiques atteignent leur apogée. Si I'autonomie disciplinaire de l'architecture semble être plus que jamais mise en doute à travers des recherches interdisciplinaires sur l'énergie, l'écologie, la mobilité etc., on assiste paradoxalement au retour des notions $d^{\prime}$ ' a autonomie de l'architecture » et l'appel au caractère « absolu » de certaines formes architecturales, chez un théoricien comme Pier Vittorio Aureli, notamment en référence à Aldo Rossi? .

Cette recherche d'une architecture absolue et autonome peut-elle être qualifiée de simple phénomène de mode, ou bien est-ce une tendance plus profonde, exprimant un besoin d'arrêt, d'immobilisation et de suspension de la réalité ? Est-ce une réaction nostalgique et romantique : une rêverie mélancolique comme
2. Monolithe et architecture en suspens, FRAC Centre, Orléans, 18 septembre 2010-27 février 2011. II est intéressant de remarquer qu'Archilab qui, pendant la dernière décennie, se positionnait comme le défenseur d'une architecture numérique basée sur les technologies les plus innovantes, se soit tourné vers le monolithe et son caractère suspensif qui, d'un point de vue idéologique aussi bien que poétique, s'oppose au progressisme et au positivisme forcené des recherches et discours actuels autour de l'architecture computationnelle.

3. Architectures de papier (11 octobre 2012-17 mars 2013), Paris, Cité de l'architecture et du patrimoine. Rêve de monuments, une exposition entre architecture réelle et architecture imaginaire (22 novembre 2012-24 février 2013), Paris, La Conciergerie.

4. Exposition La Tendenza, Architectures italiennes, 1965-1985, Paris, Centre Georges
Pompidou (20 juin-10 septembre 2012).

5. Nous pensons ici à la méthode "typo-morphologique » qui fut largement développée en Europe et surtout en France dans les années 1980

6. Le nom de la première de ces deux revues contemporaines italiennes, San Rocco, est une référence directe au titre d'un projet commun d'Aldo Rossi et Giorgio Grassi, en 1971, à Monza, Italie. 
le suggéraient déjà Manfredo Tafuri et Georges Teyssot à propos de l'œuvre d'Aldo Rossi dans les années 1980 ? Ou bien est-ce l'expression, consciente et légitime, d'une survivance : des traces et des fantômes qui veulent créer des discontinuités et des seuils temporels dans l'ordre écrasant des flux continus de la mobilité ?

Notre hypothèse est que l'image survivante, monolithique, absolue et autonome, est là pour suspendre les changements incessants d'un monde régi par les avancées technologiques et les idéologies progressistes, sans pour autant prôner un retour illusoire à une tradition continue dont l'impossibilité a été démontrée depuis longtemps.

Le suspens architectural, comme expression de ce double refus, ne saurait-il faire autrement que de laisser ressurgir des images, fragments mythiques venus d'ailleurs : les copier à l'identique, ou encore les inscrire par un procédé de collage, pour exprimer le fantôme de quelque chose qui ne peut se reproduire ? Ou alors, sommes-nous en droit d'imaginer une « répétition compulsive " qui saura faire surgir une mémoire enfouie dans le temps, mais avec la fulgurance de la nouveauté ?

Afin de répondre à ces questions, notre réflexion ne prendra pas comme objet des projets qui revendiquent une forme d'inactualité, tout en tendant paradoxalement à constituer un nouveau zeitgeist dont l'enjeu serait l'immobilisation, l'isolement, l'autonomie et le suspens. Le parti pris de cet article est plutôt de s'intéresser aux prémices de ces images survivantes, donc à Aldo Rossi qui fut le premier à évoquer l'atmosphère de « suspension » propre à l'architecture, en reconsidérant son œuvre à travers le prisme du " suspens architectural ", comme un phénomène à la fois historique, esthétique et psychologique, tout en esquissant les prémices d'une théorie du suspens en architecture.

\section{De la suspension au suspens}

Dans Autobiographie scientifique, Rossi écrit : "Chacun de mes projets ou de mes écrits me semblait définitif, dans une double acceptation du terme: d'une part, ils représentaient la conclusion d'une expérience, et de l'autre j'avais l'impression qu'il n'y aurait plus rien à dire ensuite. Chaque été me semblait le dernier, et ce sentiment d'immobilité sans évolution peut expliquer nombre de mes projets ${ }^{8}$. "

Les termes «immobilité » et «suspension» apparaissent à plusieurs reprises dans Autobiographie scientifique, sans être théorisés en tant que tels. Ils désignent un état d'esprit qui guide le projet, mais permettent aussi de qualifier une atmosphère que l'on retrouve dans les dessins et dans les photographies des édifices réalisés par Rossi : les photographies du monument aux morts de Segrate, celles des cours d'écoles primaires de Broni et de Fagnano, ou encore les images du Théâtre du Monde à Venise, expriment cette atmosphère de suspension qui ne représente pas la vie au présent de l'édifice, mais cherche davantage à saisir sa potentialité à être vécue.

La première occurrence de la notion de suspension dans Autobiographie scientifique vient à la suite de l'exposé d'une impression générée par la confusion entre un mot et un objet: « Dans l'intérêt que je porte aux objets, je dois admettre qu'il m'est arrivé constamment
7. Pier Vittorio Aureli, The Project of Autonomy, Politics and Architecture within and against Capitalism, New York, Princeton Architectural Press, 2008. Voir aussi Pier Vittorio Aureli, The Possibility of an Absolute Architecture, Cambridge (Mass.), The MIT Press, 2011.
8. Aldo Rossi, Autobiographie scientifique, Marseille, Éditions Parenthèses, 1988 [éd. originale en américain, A Scientific Autobiography, Cambridge (Mass.), The MIT Press, 1981], p. 9-10. 


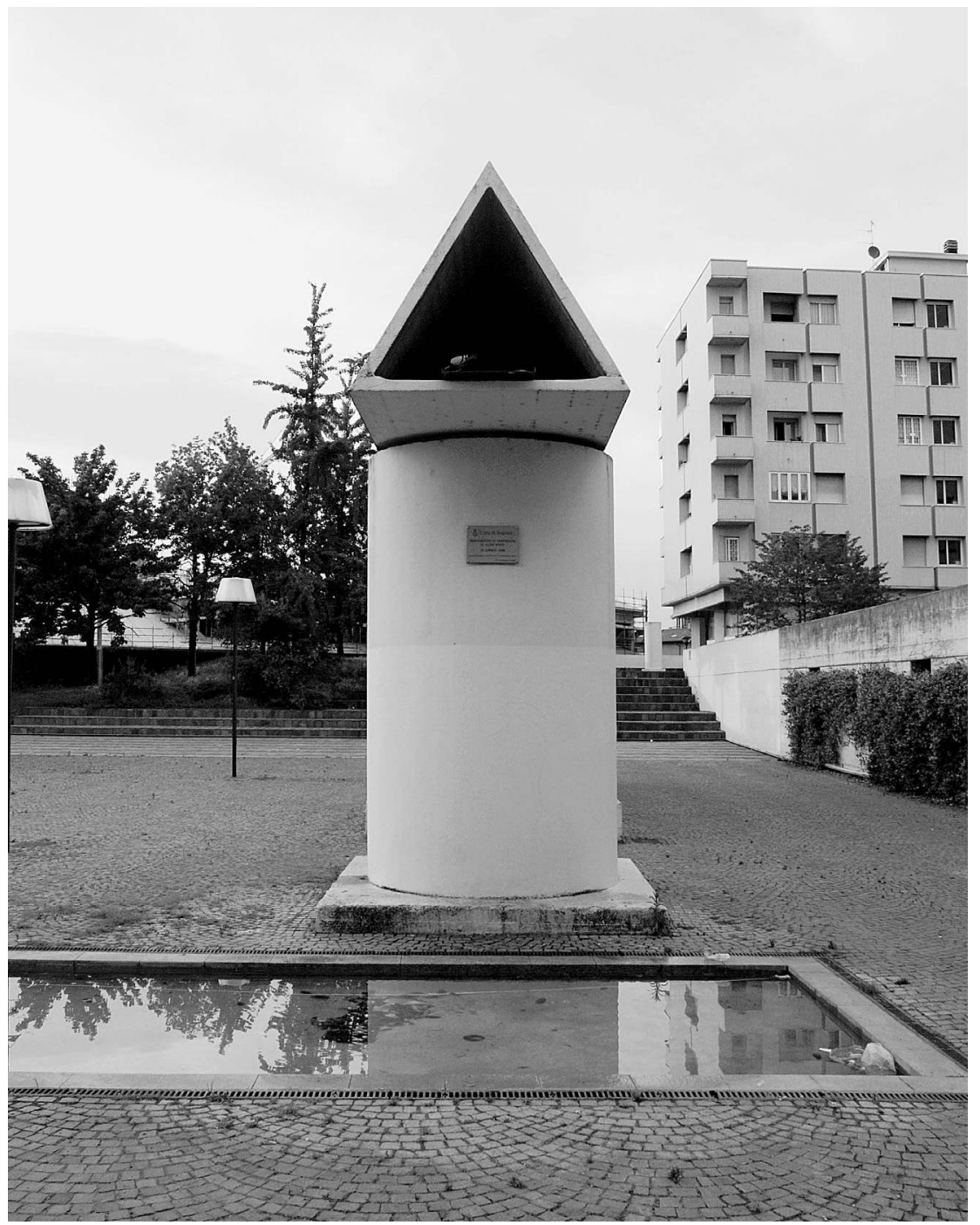

Aldo Rossi, place de la mairie et fontaine monumentale, Segrate, 1966 : vue de face. Photographie de l'auteur, 2011.
9. Ibid., p. 18.

10. Ibid.

11. Ibid., p. 19. Il est intéressant de remarquer qu'à chaque reprise, la notion de "suspension » est utilisée entre guillemets. Les guillemets ne sont pas là pour indiquer que le mot est emprunté à un autre discours, ni pour adoucir sa signification. Ils marquent plutôt la force d'indétermination qui correspond à l'idée de suspension. Ils créent un temps d'arrêt dans la lecture du texte afin d'extraire le mot de son contexte discursif en générant un autre plan de réflexion.

\section{Ibid., p. 57}

13. Rossi revient à plusieurs reprises sur ce sentiment d'immobilité et sur la distanciation qui en découle: "Une grande partie de la signification et du processus d'évolution du temps m'échappe encore aujourd'hui ; 
de confondre la chose elle-même avec le mot, par une sorte d'ignorance ou de préjugé, ou même pour ce moment de "suspension" que cela pouvait apporter à la signification d'une assertion, ou d'un dessin ${ }^{9}$ ».

L'idée de «suspension» découle ainsi d'une analogie entre un mot et un objet. Dans l'exemple donné par Rossi, il s'agit précisément d'une analogie entre un livre, le mot appareil et l'idée de la mort : « J'ai toujours eu avec le mot apparatus [appareil] un rapport pour le moins singulier : il est lié pour moi à la lecture et à la possession, lorsque j'étais encore adolescent, de l'ouvrage d'AlphonseMarie de Liguori intitulé précisément Apparecchio alla morte. Ce livre étrange, dont je conserve encore tant d'images dans la mémoire, me semblait lui-même un "appareil" en raison de son format plutôt petit et très allongé. J'avais même l'impression qu'on pouvait ne pas le lire et qu'il suffisait de le posséder en tant qu'instrument. Mais le lien entre l'appareil et la mort revenait également dans des expressions aussi quotidiennes que apparecchiare la tavola qui signifie dresser la table, la préparer, la disposer. À partir de là, j'ai commencé à regarder l'architecture comme l'instrument qui permettait le déroulement d'une chose ${ }^{10} »$.

Du titre d'un livre qui évoque la mort comme idée, il ne faut qu'un saut pour aboutir à un objet qui implique la mort comme événement, puis un deuxième saut pour obtenir «l'architecture comme l'instrument qui permettait le déroulement d'une chose ». Cette chose, comme le suggère I'analogie proposée par Rossi, ne pourrait être autre que la mort. Rien ici, du moins rien de rationnel, ne nous permet pourtant de comprendre pourquoi la mort serait pour Rossi à l'issue de ce déroulement.
Seul un procédé analogique de glissement du sens - une "suspension » de la signification des choses peut permettre cette identification de l'architecture et de la mort comme événement à venir.

L'idée de suspension prend donc ici un nouveau sens. Il ne s'agit plus uniquement de suspendre la signification d'une chose particulière, mais de donner à voir une suspension qui agit dans l'existence même du sujet : c'est-à-dire la mise en suspens de la mort et le rôle que joue l'architecture dans ce suspens.

En effet, la deuxième fois que l'idée de suspension apparaît dans le texte de Rossi, c'est pour évoquer une dénégation de la mort, "le sentiment de "suspension" du temps, des choses ", pour " échapper à la condition de celui qui passe ${ }^{11}$ ». Pour Rossi, I'architecture a une force d'immobilisation qui permet au sujet, ne serait-ce que momentanément, de prendre une distance par rapport à la mort. II s'agit là d'un sentiment primordial produit par l'architecture: " Je suis comme immobile, dans une situation de "suspension" qui peut résumer toute mon architecture, où la situation du lieu et du temps, qui semble si importante, se dissout en des gestes et des parcours habituels ${ }^{12}$ ».

L'architecture suspend ainsi l'action et rend indéterminées les notions de temps et d'espace. L'architecte devient le spectateur distancé d'une temporalité gelée où l'évolution des choses est déniée, I'histoire figée : un temps où la mort et la vie semblent être rejetées en dehors de l'expérience ${ }^{13}$. Le sujet créateur, mais aussi l'usager de l'architecture, sont dans un état contemplatif où la seule expérience qui reste à vivre est celle d'une " émotion glacée par le temps ${ }^{14}$ ». Dans cette émotion glacée où comme si le temps était une matière que j'observe de l'extérieur. L'absence de processus d'évolution dans mon travail a été la source de quelques mésaventures mais aussi de bonheurs. » Ibid., p. 70.

14. Rossi retrouve dans ce que Loos annonçait face au tumulus une définition de la suspension : "Aussi, les déclarations d'Adolf Loos, avec leur caractère biblique, me passionnaient-elles dans leur incapacité à trouver un développement ultérieur : une logique supra-historique de l'architecture. [...] Adolf Loos avait fait cette grande découverte architecturale : s'identifier à l'objet à travers l'observation et la description, sans altération, sans accommodement et finalement sans passion créative ou plutôt, avec une émotion glacée par le temps. »Ibid., p. 81.

Pour la lecture de Adolf Loos par Aldo Rossi, voir la thèse de doctorat soutenue par Can Onaner en 2011, Le suspens en architecture, Adolf Loos et Aldo Rossi, à Paris 1-Sorbonne, sous la direction de Dominique Rouillard. Voir également. "Analyzing the Masochistic Architect », Log 25, Reclaim Resi[liance]stance, New York, Anyone Corporation, été 2012, p. 89-92. et «Adolf Loos and the Masochistic Economy ", City Vision Mag n ${ }^{\circ}$ 6, My Masochism, Rome, juin 2012, p. 70-75. 
les choses semblent définitives et sans évolution, nous pourrions penser que les procédés créateurs de l'architecte se résument à la copie, à la citation, puis à l'autocitation, de façon à éradiquer toute nouveauté. Or au contraire, pour Rossi, cet état d'immobilité contient l'attente d'un événement qui viendrait contredire le caractère fini des choses. Au sein de la suspension agit une latence. La forme rituelle de l'architecture suppose une persistance du corps et de la matière à l'intérieur de la forme. II s'agit d'attendre que cette matière étouffée, l'énergie contenue dans la forme, refasse surface pour transformer l'immobilité en procédé actif.

\section{La mort contenue en latence à l'intérieur de la forme}

Au début d'Autobiographie scientifique, Rossi fait référence au livre de Max Planck, du même titre que le sien. Dans ce livre, Planck raconte I'histoire d'un bloc de pierre qu'un maçon soulève d'un violent effort jusqu'au toit d'une maison. Selon Rossi, " l'énergie dépensée ne se perdait pas mais restait emmagasinée pendant de nombreuses années, sans aucune déperdition - latente dans le bloc de pierre - jusqu'au jour où le bloc de pierre se détachait du toit, tombait sur la tête d'un passant et le tuait ${ }^{15}$ ".

Tandis que pour Planck cette anecdote concerne une loi physique, pour Rossi, l'énergie emmagasinée, latente dans la pierre, recèle la mort comme événement à venir. La mort n'est pas ici quelque chose qui s'oppose à la vie mais une énergie latente agissant au sein même de la vie : " une mort qui est en quelque sorte une combinaison d'énergie ${ }^{16}$ ». L'architecture, comme toute autre technique qui se confronte à la fixation de la matière dans la forme, contient cette combinaison d'énergie en son intérieur. Entre la mort qui est une énergie latente et la forme figée qui perdure en donnant l'illusion rassurante de l'intemporalité, se joue une dialectique fondamentale pour l'architecture et pour toute technique artistique : "Le principe de la conservation de l'énergie se confond dans chaque artiste ou technicien avec la recherche du bonheur et l'interrogation sur la mort ${ }^{17}$ ».

Par cette affirmation, Rossi nous dévoile sa double compréhension de la temporalité de l'architecture : d'une part un temps énergétique qui contient la mort en latence, et de l'autre, un temps idéal et anhistorique qui permet d'envisager le bonheur en déniant la mort. Le suspens, tel que nous l'entendons, est un phénomène qui se place à la lisière de ces deux temporalités. Le travail esthétique, celui de l'architecte et de l'artiste, consiste essentiellement à travailler l'équilibre entre ces deux temporalités : à sublimer la matérialité de la mort en suspendant l'énergie matérielle à l'intérieur de la forme, tout en donnant à voir quelque chose de cette énergie emmagasinée et de la menace qu'elle représente. C'est en ce sens que le suspens devient le temps de l'attente. Non plus une attente où il serait question d'attendre un événement, mais une attente qui se serait passé de son objet - en l'occurrence ici la mort - pour n'en garder qu'un sentiment de menace.

Dans L'attente, I'oubli, Blanchot écrit : "Quand il y a attente, il n'y a attente de rien. Dans le mouvement de l'attente, la mort cesse de pouvoir être attendue. L'attente, dans la tranquillité intime au sein de laquelle tout ce qui arrive est détourné par l'attente, ne laisse
15. Max Planck, Scientific Autobiography and Other Papers, New York, Philosphical Library, 1968.

A. Rossi, Autobiographie scientifique, op. cit. note 9, p. 10.
16. Ibid.

17. Ibid.

18. Maurice Blanchot, L'attente, I'oubli, Paris, Gallimard, 1962, p. 43. La lecture de Blanchot se base notamment sur le principe évident mais inquiétant selon lequel la mort comme événement est toujours impersonnel, car c'est précisément le moment où le sujet est aboli.
19. "Ce fut, indéniablement, mon premier contact avec l'art figuratif, et j'étais alors, comme je le suis aujourd'hui, attiré par la permanence et le naturel, par le classicisme des architectures et par le naturalisme des personnages et des objets. Le sentiment de "suspension » du temps, des choses, que j'éprouvais alors, suscitait en moi des états de froide exaltation [...]. Dans chacun de mes projets et de mes dessins, je crois que se profile l'ombre de ce naturalisme, il transcende leurs bizarreries et leurs défauts. 
pas arriver la mort comme ce qui pourrait suffire à l'attente, mais la tient en suspens, en dissolution et à tout instant dépassé par l'égalité vide de l'attente ${ }^{18}$. "

Pour Rossi, comme pour Blanchot, I'attente dans son état pur se substitue à la mort. Le suspens, comme une attente sans objet, dissout la réalité matérielle de la mort. Si Rossi préfère la répétition au changement, le rite à l'innovation, c'est pour générer ce temps propre à l'attente où la mort comme événement a laissé place au suspens esthétique. II s'agit de substituer les mécanismes « artificiels » de l'attente à la latence " naturelle » de la matière : substituer un suspens esthétique à la suspension matérielle.

$C^{\prime}$ est en ce sens que nous pouvons comprendre l'intérêt de Rossi pour « le naturalisme des personnages et des objets " dans les peintures d'Edward Hopper. Le sentiment de permanence et de naturel s'y double d'une attente qui se serait passé de son objet pour exprimer ce que Rossi décrit comme étant «l'immobilité de ces miracles hors du temps, tables dressées pour l'éternité, boissons jamais consommées, choses qui ne sont qu'elles-mêmes ${ }^{19}$ ». Le rôle spécifique de l'art et de l'architecture est alors de retenir l'événement par son univers esthétique : figer le mouvement, geler l'énergie contenue, mais rendre possible son émergence.

Ce qui importe ici est davantage le désir, l'attente de l'événement que l'événement lui-même, qui d'ailleurs n'adviendra peut-être jamais : «Ainsi, l'outil architectural devient le véhicule qui favorise l'émergence d'un événement que nous souhaitons, indépendamment du fait qu'il survienne ou non ; et dans ce désir de l'événement, il y a quelque chose de "progressif » au sens hégélien. [...] c'est dans ce sens-là que les proportions d'une table ou d'une maison ont une très grande importance ; non pas - comme le pensaient les fonctionnalistes - pour remplir une fonction déterminée, mais pour permettre davantage de fonctions. En fin de compte pour rendre possible ce qui dans la vie est imprévisible ${ }^{20} »$. Rossi est intéressé par la prédisposition, " par ce qui peut arriver l'espace d'une nuit d'été... Dans ce sens l'architecture peut être belle avant même son usage : c'est l'attente, la chambre nuptiale préparée, ce sont les fleurs, le ciboire d'argent avant la grande messe ${ }^{21} »$.

Tel un désir qui n'aurait plus besoin d'objet pour désirer, l'attente, quand elle s'est libérée de son objet, gagne toute sa puissance, devient autonome : elle génère son espace-temps propre qui est celui du suspens. S'il existe un "progrès », celui-ci tient du désir d'événement et de sa possibilité. Le progrès existe indépendamment du fait que cet événement se réalise ou non. La nouveauté n'appartient pas au fait historique ; il est de l'ordre du devenir suspendu.

\section{Répéter pour oublier, oublier pour répéter}

Qu'est-ce qui permet de libérer l'attente de son objet pour atteindre cet état de suspens ? La réponse est suggérée par Blanchot : il faut oublier l'objet qui était à l'origine de l'attente.

L'oubli devient un thème prépondérant dans les écrits de Rossi dès les années 1970, notamment dans Autobiographie scientifique qu'il hésite à titrer : Oublier I'architecture ${ }^{22}$. Pour Rossi, le souvenir et l'oubli sont les deux possibilités de l'architecture, qui « pour atteindre
Lorsqu'à New York j'eus l'occasion de voir l'ensemble de l'œurre d'Edward Hopper, je réalisai combien mon architecture portait cette empreinte : des tableaux comme Chair Car ou Four Lane Road me renvoyaient à l'immobilité de ces miracles hors du temps, tables dressées pour l'éternité, boissons jamais consommées, choses qui ne sont qu'elles-mêmes. ": A. Rossi, Autobiographie scientifique, op. cit. note 9, p. 19. II n'est pas anodin que Rossi fasse référence aux peintures d'Edward
Hopper dans lesquelles le suspens comme procédé esthétique est obtenu grâce à des mises en scène architecturales en jouant de la lumière et de la visibilité entre l'intérieur et l'extérieur. Nous verrons plus loin que la dialectique de la visibilité qui en découle est un procédé spécifique à l'architecture

- au travail de l'espace - auquel empruntent aussi la littérature et le cinéma pour constituer leur propre esthétique du suspens.
20. A. Rossi, Autobiographie scientifique, op. cit. note 9, p. 18.

21. Ibid., p. 122.

22. "Comme je l'ai dit, j'aurais pu tout aussi bien intituler ce livre Oublier l'architecture. Car même si j'y parle d'une école, d'un cimetière, d'un théâtre, il est plus juste de dire que je parle de la vie, de la mort de l'imagination. » Ibid., p. 145. 
une certaine grandeur, doit être oubliée, ou proposer simplement une image de référence qui se confonde avec le souvenir ${ }^{23} »$.

Que signifie ici « oublier l'architecture »? Du point de vue des sciences cognitives, l'oubli serait, comme le souvenir, une fonction de la mémoire. Il pourrait être considéré comme étant le mode de sélection qui permet au souvenir de se distinguer parmi les informations enregistrées par la mémoire. L'oubli, en ce sens, ne serait que le négatif du souvenir, ce dernier étant alors la seule information à prendre en compte.

Or nous pouvons aussi penser qu'il existe une fonction autonome à l'oubli, indépendamment de son rapport au souvenir. Selon une logique psychanalytique, l'oubli serait un mode de refoulement qui aurait sa propre économie psychologique dans la mesure où il permettrait à l'organisme de passer sous silence des affects, des sensations et des désirs qui pourraient nuire à son fonctionnement.

Dans ce cas, de l'oubli et du souvenir découleraient deux modes de répétition différents : si nous nous souvenons d'un événement qui nous a affectés, la manière dont nous allons réagir face à sa résurgence laissera une certaine place à la transformation de l'événement premier dans la mesure où la répétition serait consciente. Au contraire, I'oubli ou le refoulement de cet événement originel, parce qu'il est trop douloureux ou traumatisant, participeront d'une répétition inconsciente qui sera compulsive et pathologique ${ }^{24}$.

Nous retrouvons dans l'architecture de Rossi ces deux fonctions jouées par la mémoire. D'une part, la mémoire est le moteur de sélection qui permet à l'image-souvenir de se constituer et de durer dans le temps; de l'autre, elle agit comme une puissance de refoulement permettant à l'événement à l'origine du souvenir de se faire oublier.

La typologie, chère à Rossi, joue un rôle important dans ce double processus de souvenir et d'oubli. Pour Rossi, « oublier l'architecture, ou toute autre forme de proposition, était l'objectif d'un choix typologique invariable ${ }^{25}$ ». La répétition propre à la typologie a donc l'oubli comme raison intime. Nous pouvons alors comprendre la répétition typologique comme le mécanisme qui fait lien entre ce qui est passé dans l'oubli et ce dont on se souvient : tout en constituant une mémoire vérifiable à travers des schémas génériques, elle permettrait le refoulement des images inconscientes. D'une part, grâce à son caractère rituel, elle permettrait la constitution d'une mémoire collective transmissible à travers des signes ; de l'autre, parce qu'en elle agit une répétition inconsciente et aveugle, elle mettrait sous silence la ruine attendue de l'architecture. En évoquant l'ensemble de ses projets, Rossi affirme les regarder « à la lumière du non-achèvement, de l'abandon, ou en poursuivant la manifestation inattendue d'un nouvel événement ». II précise que "l'événement constitue la nouveauté de la chose ${ }^{26}$ ». Les notions d'abandon et de non achèvement recoupent ici celle d'oubli. Que s'agit-il au juste d'oublier ou d'abandonner? Et comment cet oubli peut-il donner lieu à un nouvel événement?

Prenons l'exemple du tombeau. L'événement à l'origine du tombeau est la mort ; du tombeau au temple, du temple au fronton, du fronton à la cabine de plage, le signe se déplace, jusqu'à ce que l'on oublie l'événement qui était à son origine. Ainsi, la répétition
23. A. Rossi, Ibid., p. 83

24. En effet, la psychanalyse nous montre que l'on répète d'autant plus que l'on oublie, la reprise étant d'autant plus compulsive que le refoulement est profond et l'événement refoulé traumatique.
25. Rossi compare la typologie invariable à « des constructions picturales et graphiques où la graphie se confondait avec l'écriture, comme une forme plus noble de graphomanie où le signe relève indifféremment du dessin ou de l'écriture. » A. Rossi, Autobiographie scientifique, op. cit. note 9, p. 80.

La typologie s'apparente alors à une grammaire où les signes agissent d'avantages comme des formes graphiques dont le contenu peut être vidé, perdant de cette manière leur fonction significative. Les formes, ou plutôt les signes, se répètent comme des masques vides, sans contenus, sans signification fixe. L'architecture gagne alors en force symbolique et en théâtralité ce qu'elle perd en signification historique ou culturelle.

26. A. Rossi, ibid., p. 104. 
Aldo Rossi, cimetière

de Modène,

1971-1978. Cabines

de plage en guise

de tombeaux.

Photographie de

I'auteur, 2011.

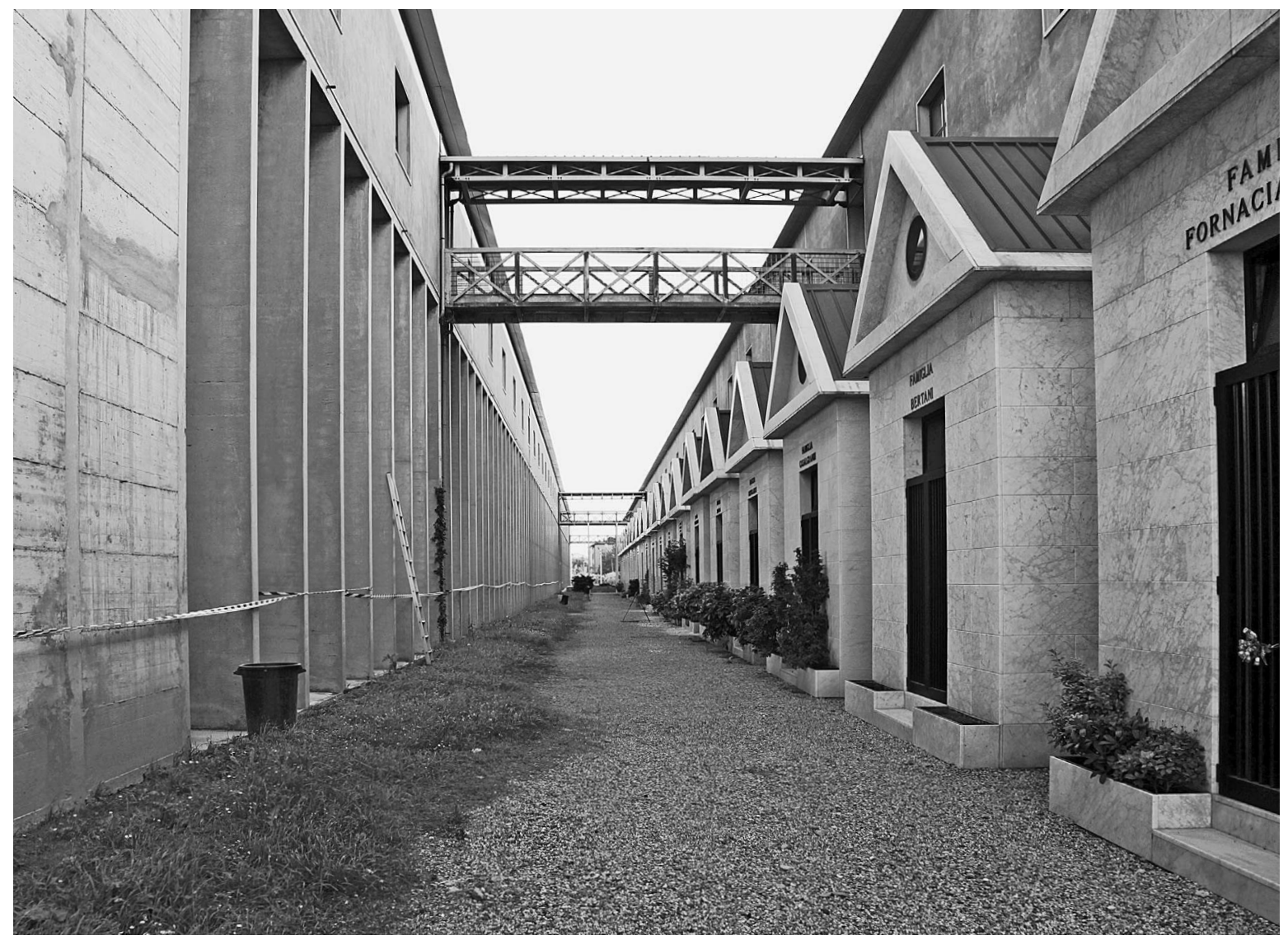


du signe «tombeau » fait oublier la mort. Mais il s'agit là d'une répétition singulière dont le but est de faire ressurgir la mort au moment voulu, car la répétition anticipe la résurgence de ce qui a été oublié : "la fenêtre, comme le cercueil, évoquent des histoires extraordinaires; mais d'un point de vue constructif, ils se ressemblent. Comme se ressemblent la tombe ou l'immeuble, car tous anticipent un événement qui est déjà arrivé quelque part, ici ou ailleurs ${ }^{27} »$.

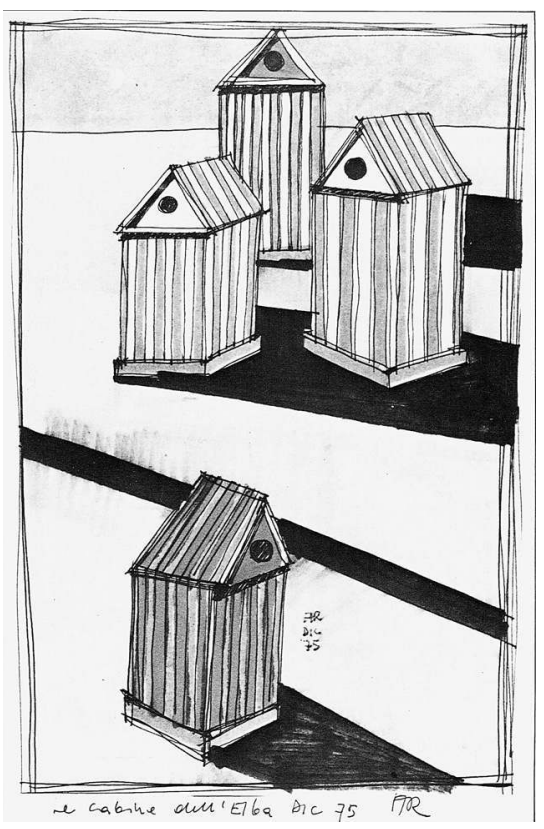

27. Ibid., p. 82.

Aldo Rossi, cabines

d'Elba, 1975. Dessin

extrait de Autobiographie

scientifique, Marseille,

Parenthèses, 1988

[éd. originale en américain,

A Scientific Autobiography,

Cambridge Mass.), The MIT

Press, 1981]
C'est donc la répétition compulsive de ces « signes tangibles » qui permet d'abord de faire oublier l'événement, puis ensuite de le laisser réapparaître de façon inattendue, selon le mode de l'« après-coup ». C'est de l'intérieur de la répétition analogique que surgissent les sensations refoulées, comme une énergie emmagasinée qui vient perturber la froideur de la classification typologique. Telle est la dualité du principe "typo-analogique » agissant au sein de la mémoire individuelle : la typologie catalogue l'objet observé pour assurer la continuité logique tout en faisant oublier l'événement à l'origine, tandis que les agencements analogiques permettent au refoulé de ressurgir dans le présent pour conjurer cette continuité et créer des associations nouvelles. C'est ainsi que la cabine de plage, signe d'un érotisme adolescent cher à Rossi, ressurgit à l'intérieur du cimetière de Modène en guise de tombeau familial, et fait remonter à la conscience l'analogie entre l'érotisme et la mort.

Parler de "nouveauté » prête donc à confusion puisqu'il s'agit en réalité de la réapparition de quelque chose qui existe depuis toujours, contenue en latence. La "nouveauté », telle que la conçoit Rossi n'est pas le fruit d'une évolution naturelle et signifiante des souvenirs. II s'agit du retour de quelque chose de familier qui apparaît de la façon la plus étrange possible. L'apparition "nouvelle » est un événement inattendu qui surgit de la mémoire et fait bloc dans le présent, empêche la signification.

C'est un enseignement de la psychanalyse qui n'échappe pas à Rossi : on n'oublie pas pour repartir à zéro ; on oublie pour répéter compulsivement la chose
Aldo Rossi, cimetière de Modène,

1971-1978. Vue depuis les fenêtres de la « maison des morts"

Collage-photographies de l'auteur, 2011. 

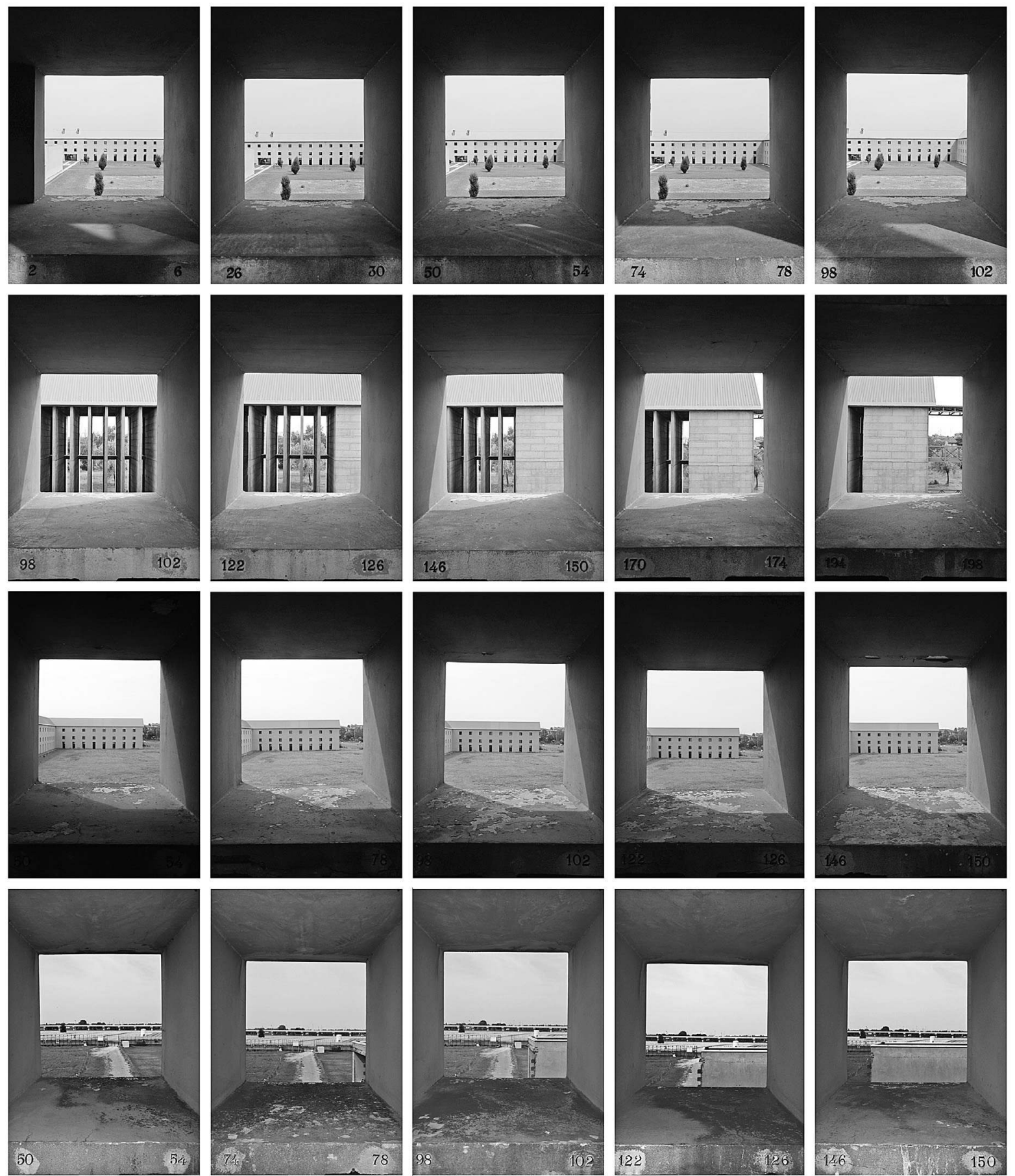
oubliée. C'est la chose oubliée qui se réitère, la même chose qui recommence, à peu de chose près. C'est en ce sens que pour Rossi «l'abandon constitue le commencement du projet, où l'abandon s'identifie à l'espoir ${ }^{28} »$.

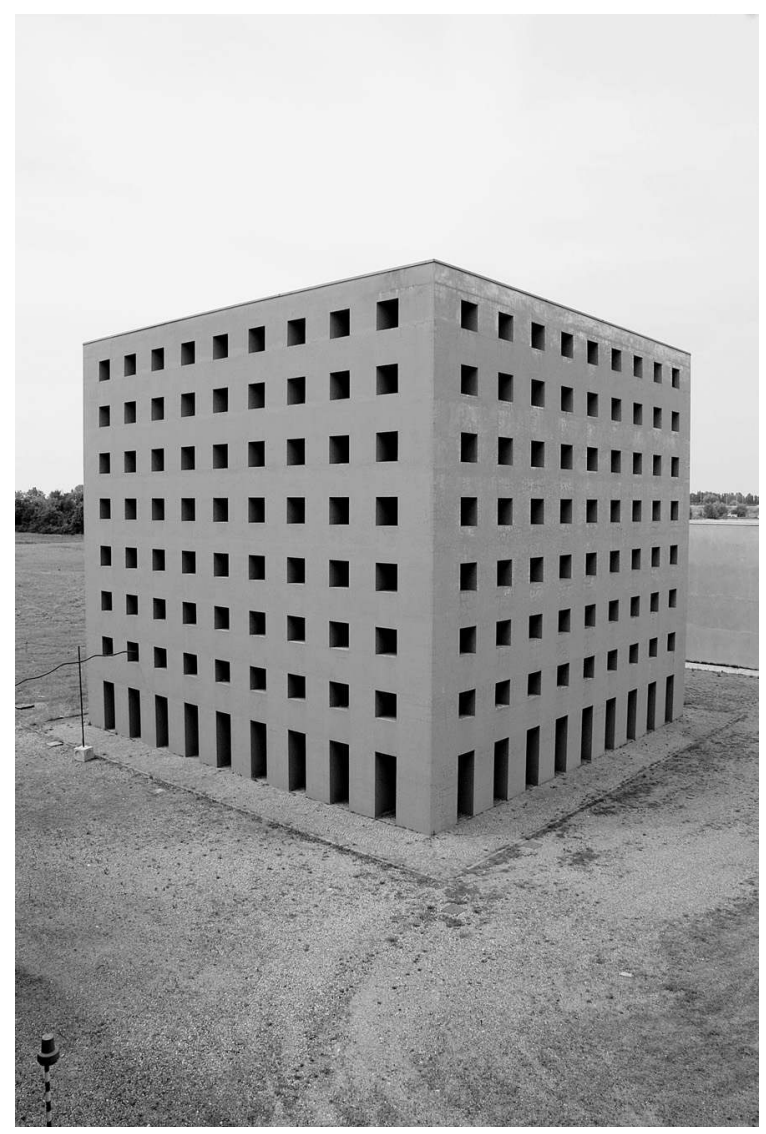

Mais il s'agit ici de l'espoir de refaire la même chose, afin de rendre cette chose plus intense encore. De la même manière qu'un amoureux reprend une même histoire d'amour qui est restée inachevée avec l'espoir de retrouver la même intensité, l'architecte réitère sans cesse un même projet qu'il a abandonné. II ne s'agit pas de refaire pour changer quelque chose, attitude qui pour Rossi relève de la superficialité, " mais de refaire à cause d'un étrange sentiment de la profondeur des choses, pour découvrir quelle sorte d'action pourrait se dérouler dans le même contexte, ou vice versa, comment le contexte, avec de légères altérations, modifierait l'action ${ }^{29}$ ".

On se retrouve ainsi devant un fonctionnement en boucle : on répète pour oublier, autant que l'on oublie pour répéter. Si la typologie invariante permettait d'oublier l'architecture, l'analogie permet de répéter en retour en déplaçant la signification de la chose répétée. Si l'on répète pour oublier, et si l'on oublie pour répéter, la chose répétée advient toujours avec une différence : "L'envie de refaire c'est un peu comme prendre deux fois la même photographie : aucune technique n'est suffisamment parfaite pour empêcher les variations dues à l'objectif, à la lumière, et finalement c'est aussi le désir de voir surgir un objet différent. Sûrement différent ${ }^{30}$ ".

Cette différence est bien plus qu'une simple variation formelle ou un changement extérieur visible dans l'objet. Le cercueil, la cabine, la fenêtre et la cheminée, sont des signes récurrents dans l'architecture de Rossi. Chaque fois qu'ils réapparaissent, la «nouveauté » réside moins dans les variations formelles propres au signe répété que dans la conscience de celui - l'architecte en premier qui contemple les résurgences du signe.
Aldo Rossi, cimetière de Modène, 1971-1978. La " maison des morts ". Photographie de l'auteur, 2011.
28. Ibid., p. 96.

29. Ibid, p. 145.

30. Ibid.
31. Gilles Deleuze, Différence et répétition, Paris, Presse universitaire de France, 1968, p. 96.

32. Ibid, p. 7. Pour Deleuze, la répétition archétypale n'est pas une "vraie » répétition, dans la mesure où elle réitère une représentation idéale et fait appel à une structure générique qui en serait le fondement. La manière dont nous entendons ici la répétition du "type ", c'est-à-dire comme la répétition d'un signe singulier, s'oppose à 
Pour expliquer ce phénomène, Gilles Deleuze cite la thèse célèbre de Hume selon laquelle « la répétition ne change rien dans l'objet qui se répète, mais elle change quelque chose dans l'esprit qui la contemple ${ }^{31} »$. Selon Deleuze, « répéter, c'est se comporter, mais par rapport à quelque chose d'unique ou de singulier, qui n'a pas de semblable ou d'équivalent. Et peut-être cette répétition comme conduite externe fait-elle écho pour son compte à une vibration plus secrète, à une répétition intérieure et plus profonde, dans le singulier qui l'anime ${ }^{32} »$.

Dans sa répétition, le type devenu image singulière donne lieu à des formes visibles indentifiables en tant que variations, mais ce qui motive véritablement la répétition est toujours cet " étrange sentiment de la profondeur des choses " qui se rapporte à l'intériorité d'une réitération silencieuse et invisible.

Quand Aldo Rossi dit à propos d'Adolf Loos, que « si rien ne peut changer, il peut rendre les choses merveilleusement plus intenses ${ }^{33}$ », il dit l'essentiel. Telle est aussi la signification que nous voudrions donner au suspens architectural : I'invention et la nouveauté ne sont pas à considérer au sein d'une évolution chronologique et selon une conception évolutionniste de la culture, mais comme la possibilité d'un événement qui est inscrit à l'intérieur de chaque chose. La nouveauté n'est pas une instance extérieure - historique, esthétique ou morale - qui s'imposerait à l'architecture mais une potentialité qui lui est immanente. Elle est inscrite dans l'histoire comme un possible tenu en suspens par I'oubli et la répétition. La nouveauté se pense alors en termes de devenir suspendu et non comme élaboration

l'idée d'archétype. Le type rossien suggère l'existence d'une dynamique intensive, différentielle, qui dirige la répétition, et non pas une structure extérieure et universelle.

33. A. Rossi, "The Architecture of Adolf Loos " (préface) dans Benedetto Gravagnuolo, Adolf Loos, Theory and Works Londres, Art Data, 1995 [première édition, New York, Rizzoli, 1982], p. 14-15. formelle ou stylistique. Elle correspond à la fulgurance avec laquelle la mémoire enfouie et oubliée va resurgir dans le présent.

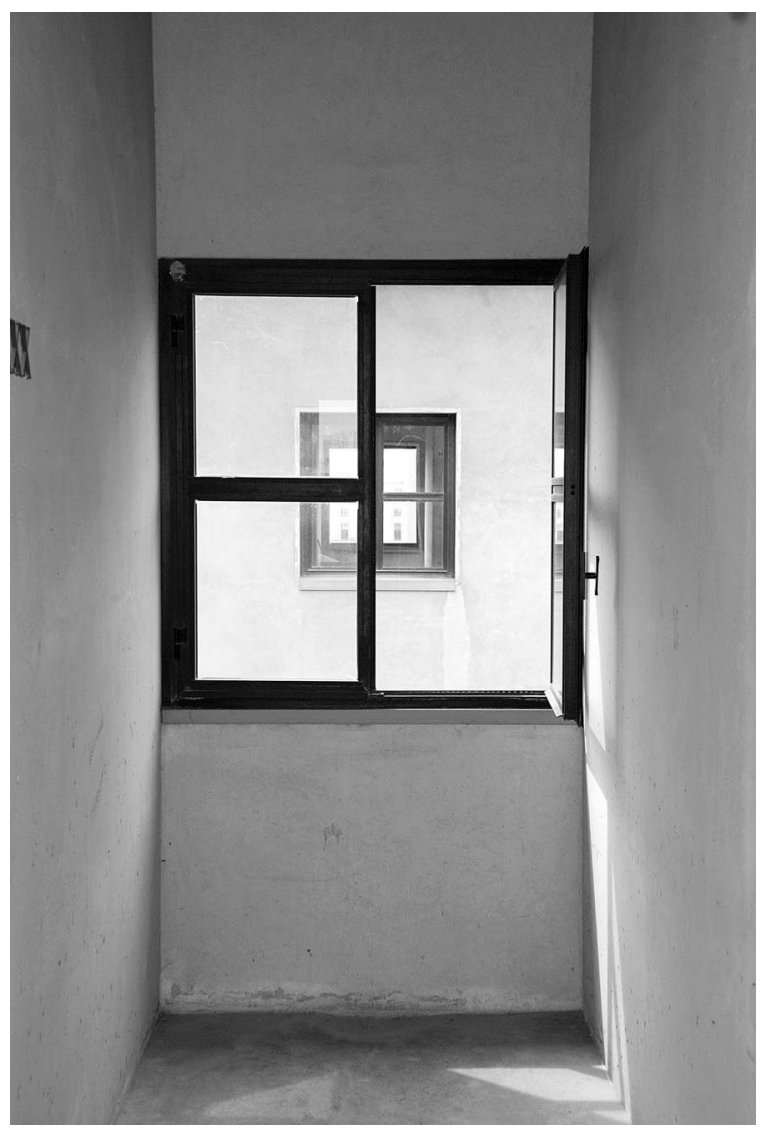

Aldo Rossi, cimetière de Modène, 1971-1978. Enfilade de fenêtres.

Photographie de l'auteur, 2011. 


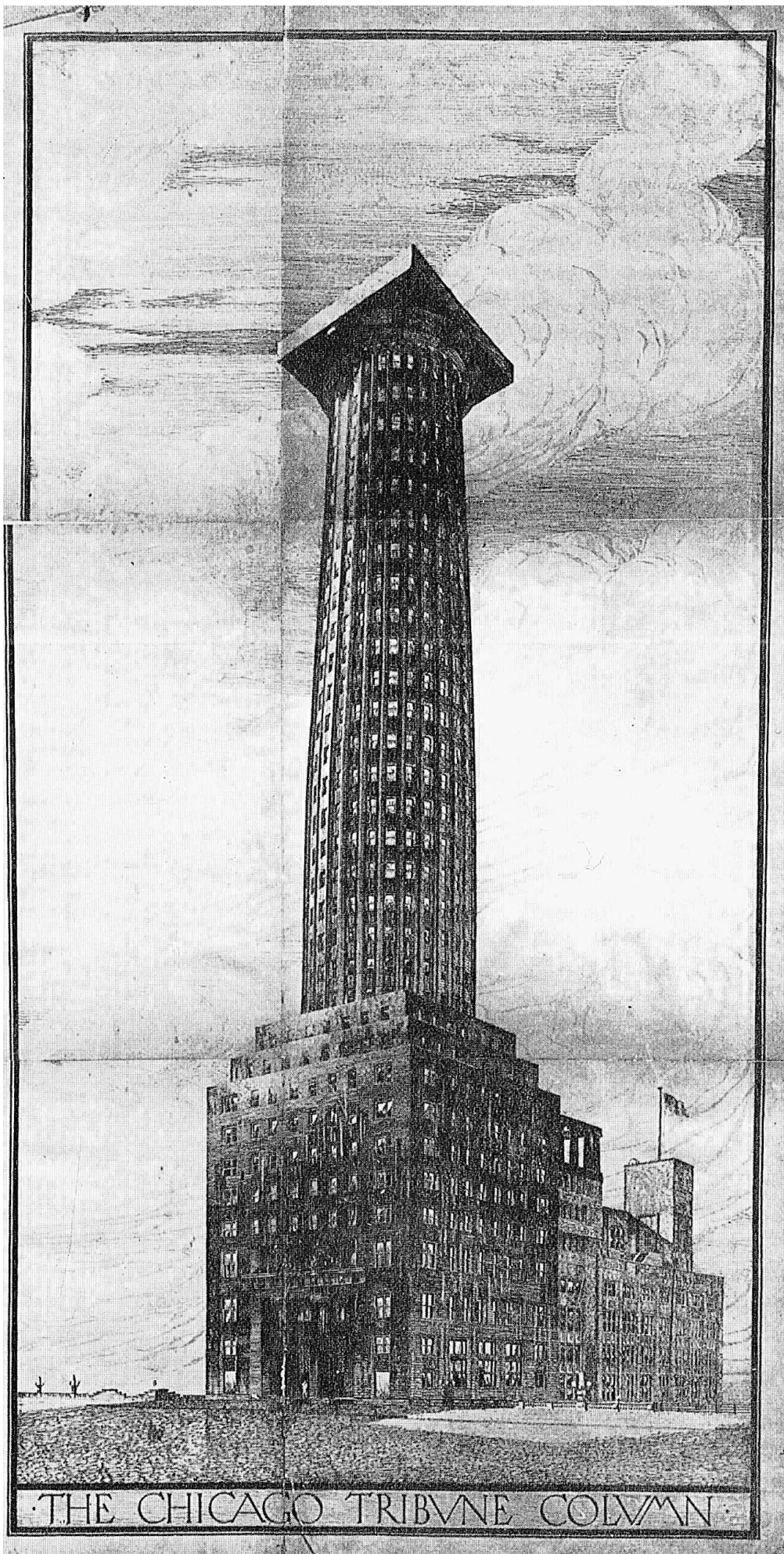

Adolf Loos, The Chicago Tribune Column, 1922. Dessin perspective dans Burkhardt Rukschcio, Roland Schachel, La vie et I'œuvre de Adolf Loos, Bruxelles, Pierre Mardaga, 1982. 